\title{
Small reductions in corolla size and pollen: ovule ratio, but no changes in flower shape in selfing populations of the North American Arabidopsis lyrata
}

\author{
Samuel Carleial $^{1} \cdot$ Mark van Kleunen $^{1} \cdot$ Marc Stift $^{1}$
}

\begin{abstract}
The shift from outcrossing to selfing is often accompanied by striking changes in floral morphology towards a "selfing syndrome", which is characterized by flowers with reduction in size, pollen: ovule $(\mathrm{P} / \mathrm{O})$ ratio, and herkogamy. This study aims to test whether such changes have occurred in the North American Arabidopsis lyrata, which is of particular interest because of the relatively recent transitions to selfing in this system. Flower size, flower shape, herkogamy levels, P/O ratio, and floral integration of six self-incompatible (outcrossing) and six self-compatible (selfing) populations of A. lyrata were measured in a common environment using conventional and geometric morphometrics methods. Although selfers had on average $9.2 \%$ smaller corollas, $8.4 \%$ longer pistils, and $21.5 \%$ lower $\mathrm{P} / \mathrm{O}$ ratios than outcrossers, there were no differences in shape, floral integration, and herkogamy between outcrossing and selfing populations. Moreover, most variation in floral traits was explained by population genetic background rather than by mating system. We conclude that selfing populations in A. lyrata have not evolved a selfing syndrome.
\end{abstract}

Keywords Breeding system $\cdot$ Evolution $\cdot$ Geometric morphometrics $\cdot$ Self-compatibility $\cdot$ Selfing syndrome

\section{Introduction}

Self-incompatibility is reported over 100 plant families, and about half of all flowering plants possess a molecular self-incompatibility system (Raduski et al. 2012). At the same time, the breakdown of self-incompatibility is very frequent (Igic et al. 2008). This has repeatedly given rise to the rapid evolution of self-fertilising (selfing) species, many of which exhibit the typical selfing syndrome, characterized by relatively small and inconspicuous flowers, reduced herkogamy, and reduced pollen:ovule $(\mathrm{P} / \mathrm{O})$ ratios (reviewed in Sicard and Lenhard 2011). For example, the selfing Capsella rubella (Brassicaceae) evolved from its outcrossing ancestor $C$. grandiflora c. 30,000-50,000 years ago (Foxe et al. 2009; Guo et al. 2009), and this was sufficient to lead to an $84.7 \%$ reduction in petal area (Sicard et al. 2011). The evolution of the selfing syndrome is not merely a neutral degeneration of traits that promote outcrossing, but also involves adaptations to the specific life history of selfers (Shimizu and Tsuchimatsu 2015). This makes the evolution of the selfing syndrome an important and interesting phenomenon both from an ecological and evolutionary perspective.

Besides directional trait changes towards the selfing syndrome, the evolution of selfing may also reduce floral integration (i.e., phenotypic correlations among floral traits; Pérez et al. 2007). The idea here is that in obligately outcrossing, self-incompatible plants, pollinators exert stabilizing selection on certain combinations of floral traits (Berg 1960). If pollinators are no longer required for pollination (with autonomous selfing), one would expect relaxed selection on these traits and, as a consequence, reduced floral integration. Indeed, reduced floral integration was reported in the selfing Leavenworthia alabamica (Brassicaceae) 
(Anderson and Busch 2006). However, we are unaware of other systems in which floral integration has been assessed after the transition to selfing, and so the generality of this pattern remains to be tested.

To allow inferences about the sequence and timing of the transition to selfing, reduction in flower size, and reduction in $\mathrm{P} / \mathrm{O}$ ratio, and avoid the confounding effects that emerge after different divergence times, study systems are needed in which the breakdown of self-incompatibility and the evolution of selfing have been relatively recent. Several such systems have been discovered in different families and studied in the context of floral morphology. For example, self-incompatibility has broken down in marginal populations associated with higher selfing rates and floral changes towards the selfing syndrome in Abronia umbellata (Nyctaginaceae) (Doubleday et al. 2013), Arabis alpina (Brassicaceae) (Tedder et al. 2015), and Camissoniopsis cheiranthifolia (Onagraceae) (Button et al. 2012). In Leavenworthia crassa, self-incompatible and self-compatible individuals occur sympatrically, but can clearly be distinguished based on flower size: selfers have smaller flowers (Lyons and Antonovics 1991). In L. alabamica, two transitions to selfing have occurred: one c. 150,000 years ago, which led to a clear flower size reduction, and one 12,00050,000 years ago, without clear size reduction (Busch et al. 2011). Hence, in most examples of intraspecific mating system variation, outcrossing and selfing populationswhile considered monospecific - are geographically separated and/or occur in distinct habitats, with distinct floral syndromes. Thus, some differences in flower morphology may not be due to selection for selfing but rather attributed to a separate evolutionary history.

To limit such confounding effects and to specifically allow making inferences about the order and timing of trait changes after the transition to selfing, it would, therefore, be useful to identify systems in which the transition to selfing is likely even more recent. A. lyrata (Brassicaceae) provides such a system. This species is normally self-incompatible, but self-incompatibility has repeatedly broken down in populations of the North American $A$. lyrata subsp. lyrata (Mable et al. 2005; Mable and Adam 2007). Most populations still have a high frequency of self-incompatible plants, and are predominantly outcrossing (multi-locus outcrossing rate: $0.83<T_{\mathrm{m}}<0.99$ ). In some populations, the majority of plants are self-compatible, and although these could theoretically still outcross, population level outcrossing rates indicate that they are predominantly selfing (multi-locus outcrossing rate: $\left.0.09<T_{\mathrm{m}}<0.41\right)$ in different population genetic backgrounds (Hoebe et al. 2009; Foxe et al. 2010; Griffin and Willi 2014). This suggests that there are multiple independent origins of selfing, and that selfing populations are of such recent origin that they are genetically very close to their putatively ancestral self-incompatible (outcrossing) populations (Foxe et al. 2010). Since selfing syndromes can evolve on relatively short timescales (e.g., 30,000-50,000 years was sufficient for $C$. rubella to develop a typical selfing syndrome; Foxe et al. 2009; Guo et al. 2009), we expect that intraspecific changes towards the selfing syndrome may already be detectible within $A$. lyrata, but should be of smaller magnitude compared to those in $C$. rubella. Alternatively, if pollinators are still required for (self) pollination, there may be no changes at all. A pilot study of floral size in A. lyrata found no consistent differences between two outcrossing and two selfing populations (Hoebe 2009), but there has not been a more conclusive comparison of floral size and morphology between outcrossing and selfing populations in this system. This is not only of fundamental interest, but may also provide important background knowledge for studies that address the ecological drivers of changes towards the selfing syndrome.

Here, we present a detailed description of the standing phenotypic variation for a suite of floral traits in outcrossing and selfing populations of A. lyrata, to test whether floral morphology in selfing populations has evolved towards states typical of the selfing syndrome and whether preadaptation may have played a role in the transition to selfing. In addition, as there are indications that corolla shape in outcrossing Brassicaceae is under strong pollinator selection (Gómez et al. 2006, 2008), we test whether there are shape changes and reduced floral integration in the selfing populations of $A$. lyrata. Although plants in these selfing populations are not entirely autonomously selfing, different pollinators may be responsible for self-pollination than for cross pollination, for which syrphid flies and small bees are assumed to be responsible (Clauss and Koch 2006; Sandring and Ågren 2009). To the best of our knowledge, potential changes in corolla shape have never been considered before in the context of the evolution of selfing. To this end, we apply landmark-based geometric morphometric analysis, which allows detecting subtle differences in floral morphology (Savriama and Klingenberg 2011). In addition, to test for reduced floral integration in selfing populations, we calculated phenotypic integration indices based on floral traits (Wagner 1984; Cheverud et al. 1989). We specifically address four questions: (1) which, if any, floral traits evolved towards states characteristic of the selfing syndrome in selfing populations, and do certain traits show larger magnitudes of change?; (2) are there differences in corolla shape between outcrossers and selfers?; (3) are flowers in outcrossing populations more integrated than flowers in selfing populations?; and (4) are differences in floral morphology between outcrossing and selfing populations consistent across different population genetic backgrounds? 


\section{Materials and methods}

\section{Study species and seed material}

Arabidopsis lyrata subsp. lyrata (Brassicaceae) grows in sand dunes, by lakesides, and on rocky outcrops. Flowers have four white petals, four long and two short stamens, and a central monocarpic gynoecium with 20-36 ovules (Al-Shehbaz and O'Kane 2002). Since the first report of the breakdown of self-incompatibility and transition to selfing in North American populations of A. lyrata around the Great Lakes (Mable et al. 2005), the system has been used as a model to address a diversity of topics relevant to the evolution of selfing, including genetic and population genetic consequences of inbreeding (e.g., Foxe et al. 2010; Haudry et al. 2012; Griffin and Willi 2014), genetic load (e.g., Stift et al. 2013; Willi 2013b), sex allocation (Willi 2013a) and resistance to pathogens (Hoebe et al. 2011). However, the floral morphology and the potential evolution towards the selfing syndrome have not been investigated to date. We used A. lyrata plants raised from seeds collected in populations in the Great Lakes region of Eastern North America (kindly provided by Dr. Barbara Mable, University of Glasgow). The mating system of these populations had previously been characterized through estimation of outcrossing rates using progeny arrays and microsatellite genotyping, and their population genetic structure had been assessed based on microsatellite and sequence markers (Foxe et al. 2010). Using this prior information, we selected six outcrossing $\left(0.82<T_{\mathrm{m}}<1.00\right)$ and six selfing $\left(0.08<T_{\mathrm{m}}<0.42\right)$ populations from seven population genetic backgrounds. Two of these backgrounds included both selfing and outcrossing populations, whereas the other backgrounds consisted of either selfing or outcrossing populations. A larger scale phylogeographic study made by Griffin and Willi (2014) identified a western and eastern cluster. All populations that we used are from the western cluster, with exception of LPT, which was westeast admixed (Griffin and Willi 2014, Online Resource 1 for details).

\section{Experimental design and photography}

In March 2013, we sowed one seed of each of 190 seed families representing the six selfing and six outcrossing populations (Online Resource 1 for sample sizes). To spread the workload of the morphometric analyses, we sowed the seeds in two batches separated by four weeks (respectively, 91 and 99 seeds for batches 1 and 2). Each population was represented in both batches with six to 23 seeds. We randomly sowed the seeds into $2.5 \times 3.2 \times 11.0 \mathrm{~cm}^{3}$ cells of six QP 54 T/11 QuickPot trays (HerkuPlast Kubern GmbH, Ering/Inn, Germany) filled with $160 \mathrm{~cm}^{3}$ potting soil
(Einheitserde und Humuswerke Gebr. Patzer GmbH \& Co., Waldsiedlung, Germany). Then, we covered the trays with transparent lids and placed them in a growth chamber with $16 \mathrm{~h}$ light at $20^{\circ} \mathrm{C}$ and $8 \mathrm{~h}$ dark at $15^{\circ} \mathrm{C}$. To minimize position effects on growth and flowering, we re-randomized positions of the trays in the growth chamber weekly. Five weeks after germination, we transplanted the seedlings into $9 \times 9 \times 8 \mathrm{~cm}^{3}$ pots (Pöppelmann $\mathrm{GmbH} \&$ Co. KG, Lohne, Germany) filled with $500 \mathrm{~cm}^{3}$ of a $2: 1$ potting soil and sand mixture. When flowering started, we fertilized the plants with 1:1000 Scotts Universol ${ }^{\circledR}$ blue (Everris International B. V., Waardenburg, Netherlands) every two weeks until the end of the experiment in September 2013.

To test whether floral morphology differed between mating systems and among populations with different genetic backgrounds, we assessed the size, shape, herkogamy, and pollen and ovule numbers of two flowers per plant for all seed families (each seed family was represented by one plant; nine out of 190 plants did not flower, Online Resource 1 for details). For size and shape estimations, we placed flowers and a size standard on a black background ( $1.5 \%$ agar with $0.5 \%$ activated carbon), such that the petals and size standard lay flat on the agar surface while standardizing the orientation of the adaxial/abaxial sides of the flowers. We then made standardized photographs of the flowers in planar front view (Online Resource 2, Fig. ESM 2.1a). To allow calculation of pistil length and antherstigma distances (herkogamy), we made a second set of photographs on the same flowers. For this, we dissected the flowers and fixed them to a tube support in side view (Fig. ESM 2.1b). For all photography, we used a NIKON D7100 camera and a SIGMA $150 \mathrm{~mm}$ F2.8 APO EX DG OS macrolens. To minimize variation due to development and time of day, we only selected flowers among the first 20 produced on the main inflorescence, and took photos between 1000 and $1500 \mathrm{~h}$. We standardized flower age by selecting flowers that had fully opened, right after anther dehiscence (fresh pollen visible on anthers).

\section{Morphometric analyses: flower size, flower shape, and herkogamy}

In total, we digitised 349 flowers, from 112 plants from six outcrossing populations, and 69 plants from six selfing populations (for 13 plants, we could only sample one flower, and for the others, we sampled two flowers per plant). To estimate flower size, we calculated the corolla area of front-view pictures using ImageJ 1.47q (http:// imagej.nih.gov/ij/) and performed a more detailed assessment of size and shape using landmark-based geometric morphometrics (Online Resource 2 for details). To quantify shape variation, we first made four geometric transformations of the landmark-configuration data: identity, rotation 
by $180^{\circ}$, reflection about the vertical axis, and rotation plus reflection (modified from Savriama and Klingenberg 2011). Then, we estimated corolla shape variation with a full Procrustes fit on the transformed landmarks (averaged by plant) followed by a Principal Component Analysis (PCA) (Klingenberg 2014) using MorphoJ 1.06d (http://www. flywings.org.uk/MorphoJ_page.htm). Similar to Savriama et al. (2012), we accounted for the flower as a disymmetric (two perpendicular symmetry axes) object in our analysis (Fig. 2a, b). Hence, we categorized flower shape, i.e., corolla top-view arrangement, into four types of symmetry variation: dissymmetric, monosymmetric with top-bottom differences (termed adaxial-abaxial in Savriama et al. 2012), monosymmetric with left-right differences, and asymmetric (termed rotational asymmetry in Savriama et al. 2012). Dissymmetric variation can be interpreted as the change in corolla shape, where each of the four petals show equal magnitude of directional change, both vertically (from top to bottom) and horizontally (from left to right). In practice, this category of shape variation represents thickness and form of petals. Monosymmetric variation can be interpreted as the symmetrical change in corolla shape, given that corolla has two symmetry axes, on only one axis, whereas the other axis show mirror image mismatch. Thus, monosymmetry variation can assume two types, rather left-right match with top-bottom differences or top-bottom match with left-right differences. This type of variation may represent differences in petal position (more to one side than to the other), proportional petal area and petal form. Finally, asymmetric variation can be perceived as the lack of similarity between neighbour petals and no mirrorimage match between the halves of the horizontal or vertical axes. This category shows combined changes in rotation and reflection when you compare any two halves of the corolla. To estimate pistil length and herkogamy, we placed eight true landmarks on stamens and pistils of the same flowers used for size and shape estimations and calculated Euclidean distances between them using coordinates of the landmark data (Online Resource 2).

\section{Pollen number, ovule number, $\mathrm{P} / \mathrm{O}$ ratio, and pollen size}

To estimate $\mathrm{P} / \mathrm{O}$ ratios, we sampled two flower buds from each plant in our design that flowered and produced sufficient flowers (13 out of the 181 flowering plants stopped flowering before bud sampling, Online Resource 1 for details). For each collected bud, we counted the numbers of ovules and pollen grains. To count ovules, we first cut the ovaries lengthwise along their septum using fine forceps and razor blades. Then, we placed both halves of the ovaries on a Petri dish, and counted the ovules using a stereoscope $(60 \times$ magnification) in which the light beam was set from below (Kearns and Inouye 1993). To count pollen grains, we first collected the six non-dehisced anthers in Eppendorf tubes using fine forceps. Then, we left the tubes opened in an oven at $70{ }^{\circ} \mathrm{C}$ overnight to promote dehiscence of pollen. On the following day, we added $30 \mu \mathrm{l}$ of $5.0 \%$ Tween- 80 to each tube, then vortexed and sonicated each tube for 20 min with a SONOREX RK 156 H (BANDELIN electronic GmbH \& Co. KG, Berlin, Germany) to release pollen from the anthers. To count pollen and measure pollen size, we transferred $10 \mu \mathrm{L}$ of the Tween-pollen suspension to a Marienfeld Superior counting chamber (Paul Marienfeld GmbH \& Co. KG, Lauda-Könighofen, Germany). For each slide, we photographed five of the 16 counting chamber's sub-quadrants (each covering an area of $2500 \mu^{2}$ with a volume of $0.004 \mu \mathrm{l}$ ) using a light transmitting microscope Axioskop (Carl Zeiss Microscopy $\mathrm{GmbH}$, Germany). Finally, we estimated pollen number and pollen size using the function "Analyze Particles" in Image J $1.47 \mathrm{q}$.

\section{Floral integration}

Based on their expected functional integration to achieve pollination and fertilisation (Klingenberg 2014), we selected six floral traits to estimate the floral integration: corolla area, short-stamen length, long-stamen length, pistil length, pollen number, and ovule number. These traits correspond to those used in a similar study in Leavenworthia (Anderson and Busch 2006). As a measure of floral integration of organ size and pollen and ovule number, we first calculated phenotypic integration indices based on the variance of the eigenvalues of the correlation matrix between the phenotypic traits (Wagner 1984), then we corrected those indices by the number of traits and samples per population (Cheverud et al. 1989), using the R package PHENIX (Torices and Muñoz-Pajares 2016) in R 3.0.2 (R Core Team 2013). To test whether the corrected phenotypic integration indices differed between mating systems, we used a one-sided Mann-Whitney test.

\section{Other statistical analyses}

To test whether flower size (corolla area and centroid size, the square root of the summed squared distances of each landmark from the centroid of the landmark configuration), flower shape (Principal Component scores of shape), shortand long-stamen herkogamy, pistil length, and $\mathrm{P} / \mathrm{O}$ ratio differed between outcrossing and selfing populations, we used general linear mixed-effects models (GLMMs) in R with a Gaussian error distribution ( $R$ package lme4, Bates et al. 2014). The fixed effects included mating system (outcrossing or selfing) and population genetic cluster (based on microsatellites and sequence markers Foxe et al. 2010). To account for blocking effects and non-independence 
of multiple measurements per population and plants, we included population, tray, and plant in the random part of the model. To simplify the analysis, our models did not include batch, as initial models, including batch, did not provide a significantly better fit (data not shown). To improve normality and homogeneity of the residuals, we $\log _{\mathrm{e}}$-transformed corolla area, and we square-root-transformed corolla perimeter and herkogamy. As we were interested in how genetic clusters differed from each other, we included genetic cluster as a fixed effect. Furthermore, this allowed us to specifically test whether differences due to mating system were consistent across genetic clusters. To this end, we analysed the subset of the two genetic clusters that contained both outcrossing and selfing populations separately. For this, we used the same modelling approach as for the complete data set, with the addition of the interaction between mating system and population genetic cluster. For assessing significance of the fixed terms, we fitted the models using maximum likelihood (ML), and compared models with and without each fixed term using likelihood-ratio tests (LRTs; Zuur et al. 2013). In cases, where population genetic cluster had a significant effect, we used post hoc Tukey tests with the function glht in the R package multcomp (Hothorn et al. 2015) to assess which pairs of clusters differed significantly.

\section{Results}

\section{Flower size}

There was considerable variation in corolla area within and among populations (Fig. 1a). On average, selfers had $9.2 \%$ significantly smaller corolla area (mean $\pm \mathrm{SD} ; 53.41 \pm 10.32 \mathrm{~mm}^{2}$ ) than outcrossers $\left(58.85 \pm 11.56 \mathrm{~mm}^{2}\right)$. Centroid size (square root of the summed squared distances of each landmark from the centroid of the landmark configuration) did not differ significantly between selfing and outcrossing populations. Compared to the effect of mating system, there were more pronounced differences in both corolla area and centroid size among populations that belonged to different genetic clusters (Fig. 1a). For example, the two clusters with outcrossing and selfing populations differed: cluster "blue" (with populations PCR-PIN-PTP-RON, in which outcrossing populations are underlined) had a $30.4 \%$ larger corolla area and a $22.1 \%$ larger centroid size than cluster "pink" (TSS-TC-TSSA) (Online Resource 3 for all GLMM contrasts). Within the subset of the two clusters with outcrossing and selfing populations (clusters "blue" and "pink"), corolla area and centroid size differed significantly between clusters, but mating system and the mating system by cluster interaction did not have a significant effect (Online Resource 4).

\section{Flower shape}

The majority of total shape variation $(72.0 \%)$ was dissymmetric and mainly accounted for by PC 1 , which explained $66.4 \%$ of the total variance and represented the degree to which flowers were compressed along the two perpendicular symmetry axes. Gómez et al. (2006) found the same pattern in E. mediohispanicum, describing it as the degree to which petals were parallel. The remaining $28.0 \%$ of the total shape variation was mainly monosymmetric: the second principal component (PC 2; $13.3 \%$ of total variance) reflected top-bottom variation and the third principal component (PC 3; 10.6\%) reflected left-right variation (Fig. 2c; Online Resource 2). The other principal components (among others reflecting asymmetry), each explained less than $10.0 \%$ of the total variance.

There were no significant differences in shape between outcrossing and selfing populations for dissymmetric (PC 1) and monosymmetric (PC 2) shape variations (Fig. 3). For the left-right monosymmetric variation (PC 3), we found that flowers with petals converging to the left were more common in selfers, whereas in outcrossers, we found the opposite tendency (Table 1). Population genetic cluster explained variation in shape for the dissymmetric and left-right monosymmetric variation, which accounted for $77.0 \%$ of the total variation. Post hoc Tukey tests, however, showed that contrasts between genetic clusters were not significant for any of the shape components (Online Resource 3 ). Within the subset of the two clusters that contained both outcrossing and selfing populations (clusters "blue" and "pink"), the effects of mating system, genetic cluster and their interaction were also not significant (Online Resource 4).

\section{Stamen length, pistil length, and herkogamy}

Selfers had $8.4 \%$ longer pistils (mean \pm SD; $3.79 \pm 0.36 \mathrm{~mm})$ than outcrossers $(3.47 \pm 0.26 \mathrm{~mm}$; significant effect of mating system in Table 1), but there were no significant differences between mating systems in stamen length or herkogamy. There were significant differences among population genetic clusters for pistil and stamen length, but not for herkogamy (Table 1). Compared to cluster "blue" (populations PCR-PIN-PTP-RON), cluster "pink" (TSS-TC-TSSA) had shorter pistils (although contrast only marginally significant at $P=0.065$; Fig. 1b), significantly shorter short stamens $(2.48 \pm 0.12 \mathrm{~mm}$ vs $2.80 \pm 0.05 \mathrm{~mm}$ ) and significantly shorter long stamens (3.37 $\pm 0.14 \mathrm{~mm}$ vs $3.78 \pm 0.09 \mathrm{~mm}$ ) (Online Resource 
Fig. 1 Differences among genetic clusters in outcrossing (triangles) and selfing (filled circles) populations of Arabidopsis lyrata regarding a corolla area, b pistil length, and $\mathbf{c} \mathrm{P} / \mathrm{O}$ ratio. Values are mean \pm SEM of untransformed data. Colour codes given correspond to the colours used in Fig. 1 of Foxe et al. (2010). Sample sizes can be found in ESM 1. Significant differences between clusters (based on Tukey post hoc comparisons corrected for multiple testing) are indicated through dotted lines with the test statistic and $P$ value (ESM 3 for all GLMM contrasts and post hoc Tukey tests)
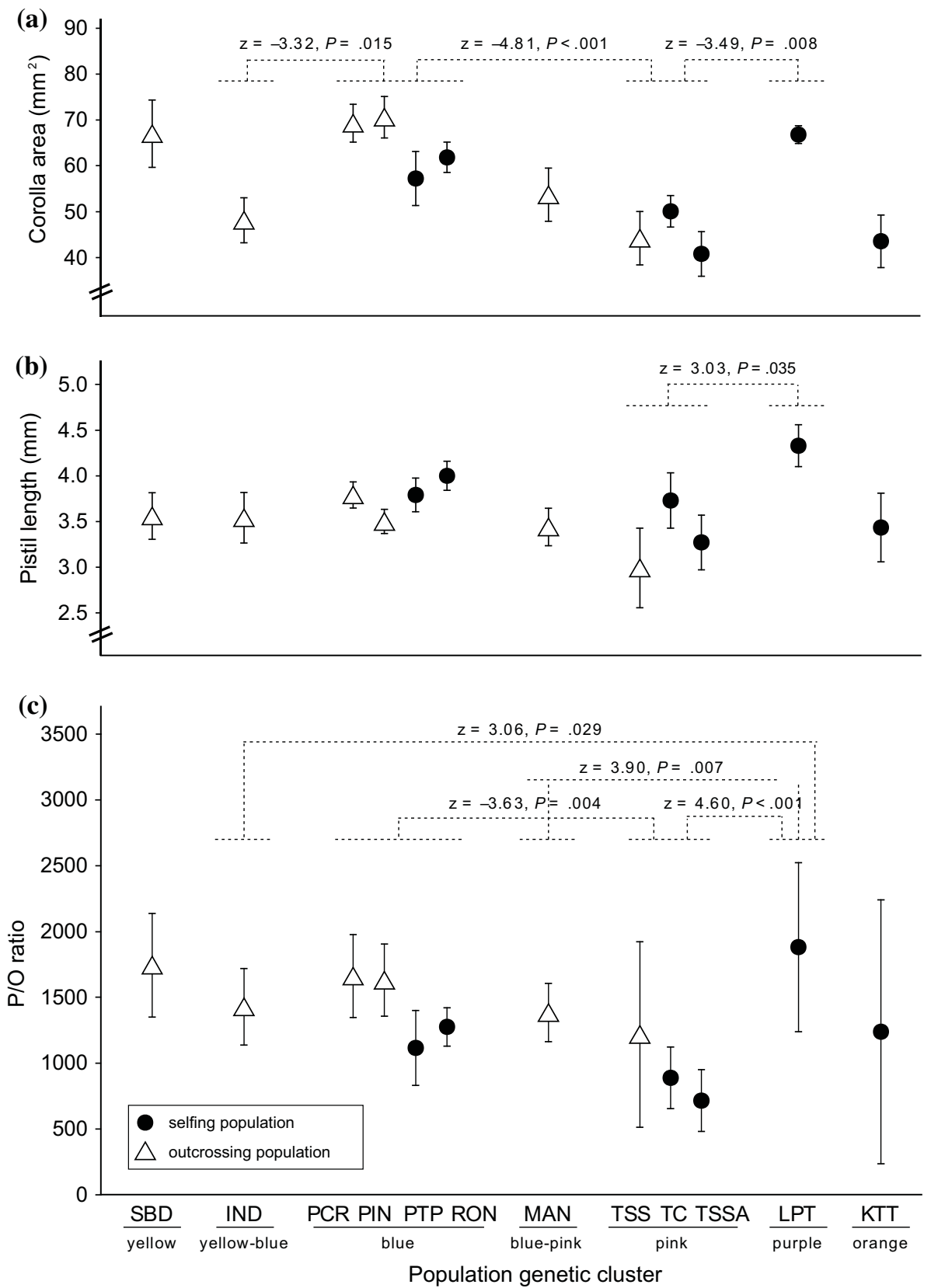

3). Within the subset of the two clusters that contained both outcrossing and selfing populations (clusters "blue" and "pink"), short and long stamen length differed significantly between clusters, but mating system and the mating system by cluster interaction did not have a significant effect (Online Resource 4).

\section{Pollen number, ovule number, $\mathrm{P} / \mathrm{O}$ ratio, and pollen size}

On average, selfers produced $9.0 \%$ fewer pollen grains (mean $\pm \mathrm{SD} ; 36,372 \pm 8,554$ ) and $12.7 \%$ more ovules $(32 \pm 5)$ than outcrossers $(39,952 \pm 5,377$ pollen grains; $28 \pm 3$ ovules; significant effects of mating system in
Table 1). This translated into a significant $21.5 \%$ reduction in $\mathrm{P} / \mathrm{O}$ ratio for selfing compared to outcrossing populations (Table 1). There were also significant differences among population genetic clusters in pollen number, ovule number, and $\mathrm{P} / \mathrm{O}$ ratio (Table 1). For example, cluster "pink" (TSS-TC-TSSA) had a significantly lower number of pollen grains than cluster "purple" (LPT), and produced more ovules and had a lower $\mathrm{P} / \mathrm{O}$ ratio than cluster "blue" (PCR-PIN-PTP-RON) (Fig. 1c; Online Resource 3). Cluster "purple" (LPT) had the highest P/O ratio (significantly higher than all clusters except "yellow" and "orange", Fig. 2). Variation in pollen size was neither explained by mating system nor by population 


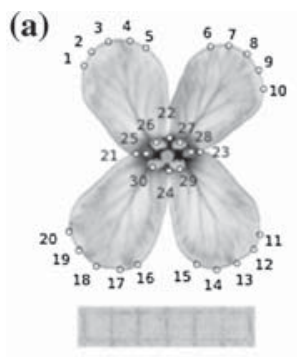

(c)

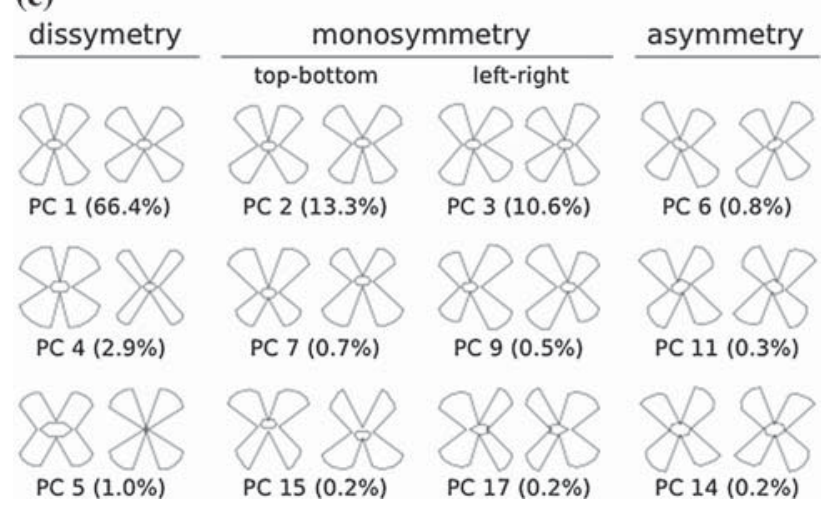

Fig. 2 Geometric morphometric analysis of flower shape in Arabidopsis lyrata. a Flower example with homologous and pseudo landmarks. Scale $6 \mathrm{~mm}$. b Flower orientation in relation to the two inflorescence symmetry axes (top-bottom and left-right). Flower diagram represents the average flower shape based on the landmark data, and individual dots are real landmarks taken from digitised flowers $(n=349)$. c Dissymmetric, monosymmetric (top-bottom or leftright) and asymmetric floral shape variation represented by principal components (PCs). Only the first three PCs per category are shown with their relative importance (percentage of explained variance), and with two schematic drawings of the -0.1 (left) and +0.1 (right) PC scores (see ESM 2 for details)

genetic cluster (Table 1). Within the subset of the two clusters with outcrossing and selfing populations (clusters "blue" and "pink"), ovule number and P/O ratio differed significantly between clusters, and between outcrossing and selfing populations, but there was no significant mating system by cluster interaction. There were no differences in pollen number and size among clusters and mating systems (Online Resource 4).

\section{Floral integration}

Phenotypic integration in A. lyrata was significant for all estimations and ranged from 0.222 (95\% CI: 0.158-1.850) to 1.151 (95\% CI: $0.664-2.040)$ in selfing populations, whereas from 0.447 (95\% CI: $0.319-1.084)$ to 1.308 (95\% CI: 0.456-2.504) in outcrossing populations (Online Resource 5). There was no significant difference in floral integration of organ size and pollen and ovule number between selfing and outcrossing populations (Mann-Whitney test, $W=20, P=0.214$ ).

\section{Discussion}

Our results indicate that population genetic structure was quantitatively more important in explaining variation in floral traits than mating system in A. lyrata. Although flowers from selfing populations were significantly smaller by $9.2 \%$ and had $21.5 \%$ lower $\mathrm{P} / \mathrm{O}$ ratios than outcrossing populations, corolla shape, stamen lengths, herkogamy, pollen size, and floral integration did not differ significantly between outcrossers and selfers. These findings suggest that in comparison with other species with intraspecific mating system variation, the evolution of selfing in $A$. lyrata has not yet led to strong changes towards the selfing syndrome. Instead, our data show that plants belonging to certain population genetic clusters possess floral traits closer to the selfing syndrome-among others, have smaller flowers-regardless of their mating system. However, pre-adaptations to the selfing syndrome have not been crucial for the evolution of selfing in A. lyrata, since selfing

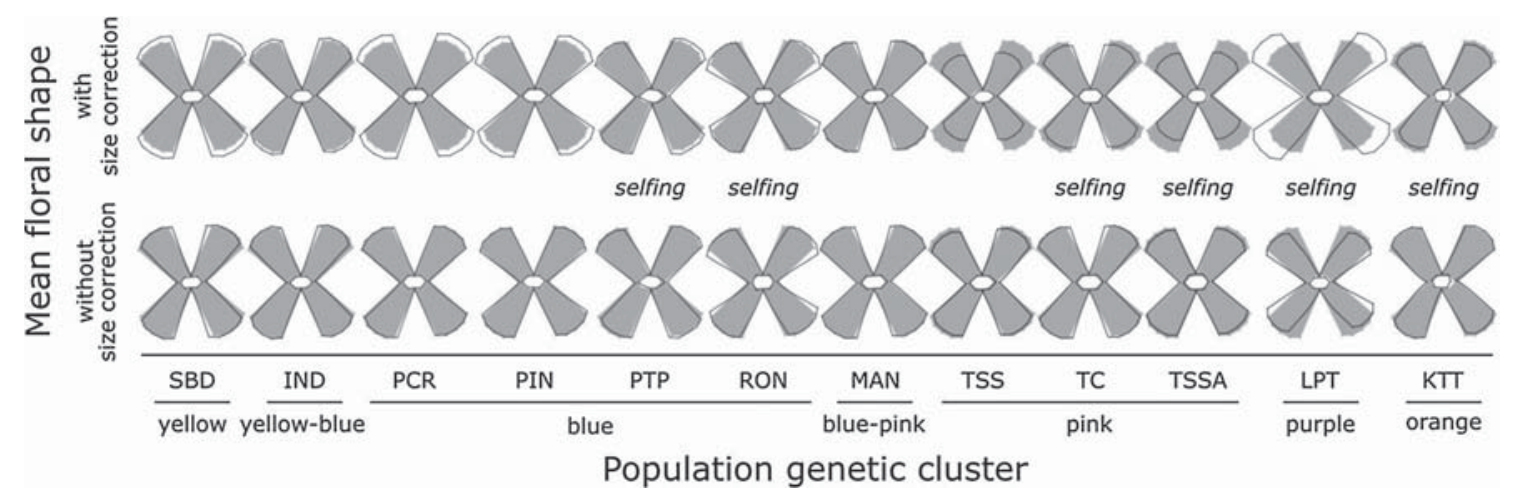

Fig. 3 Flower shape variation among populations (black outlines) grouped by genetic cluster compared to the mean shape (grey outline) with (top) and without (bottom) correction for centroid size 
Table 1 Effects of population genetic cluster and mating system on floral and reproductive traits of Arabidopsis lyrata from a general linear mixed-effect model analyses

\begin{tabular}{|c|c|c|c|c|c|c|c|c|}
\hline \multirow[t]{4}{*}{ Floral trait, (unit) } & \multicolumn{4}{|c|}{ Fixed effects } & \multirow{2}{*}{\multicolumn{4}{|c|}{$\frac{\text { Random effects }}{\text { Variance estimates }}$}} \\
\hline & \multirow{2}{*}{\multicolumn{2}{|c|}{$\begin{array}{l}\text { Population } \\
\text { genetic cluster } \\
(d f=6)\end{array}$}} & \multirow{2}{*}{\multicolumn{2}{|c|}{$\begin{array}{l}\text { Mating system } \\
(d f=1)\end{array}$}} & & & & \\
\hline & & & & & \multirow[b]{2}{*}{ Population } & \multirow[b]{2}{*}{ Plant } & \multirow[b]{2}{*}{ Tray } & \multirow[b]{2}{*}{ Residua } \\
\hline & $\chi^{2}$ & $P$ & $\chi^{2}$ & $P$ & & & & \\
\hline Corolla area, $\log _{\mathrm{e}}\left(\mathrm{mm}^{2}\right)$ & 29.15 & $<0.001$ & 3.93 & 0.047 & 0.006 & 0.031 & 0.001 & 0.027 \\
\hline Centroid size & 27.72 & $<0.001$ & 0.41 & 0.521 & 1.837 & 3.251 & 0.202 & 2.393 \\
\hline Corolla perimeter, sqrt $(\mathrm{mm})$ & 28.51 & $<0.001$ & 2.42 & 0.120 & 0.026 & 0.072 & 0.008 & 0.144 \\
\hline Dissection index, $\log _{\mathrm{e}}()$ & 25.53 & $<0.001$ & 0.03 & 0.866 & $<0.001$ & 0.004 & $<0.001$ & 0.005 \\
\hline Corolla shape, dissymmetric variation (PC 1) & 18.36 & 0.010 & 2.50 & 0.114 & 0.002 & 0.004 & 0.001 & 0.011 \\
\hline Corolla shape, monosymmetric top-bottom variation (PC 2) & 10.00 & 0.189 & 0.01 & 0.911 & $<0.001$ & $<0.001$ & $<0.001$ & 0.005 \\
\hline Corolla shape, monosymmetric left-right variation (PC 3) & 14.71 & 0.040 & 5.83 & 0.016 & $<0.001$ & $<0.001$ & $<0.001$ & 0.004 \\
\hline Pistil length, (mm) & 20.78 & 0.004 & 7.96 & 0.005 & 0.032 & 0.203 & $<0.001$ & 0.162 \\
\hline Short-stamen length, sqrt(mm) & 28.02 & $<0.001$ & 0.29 & 0.593 & 0.003 & 0.016 & $<0.001$ & 0.011 \\
\hline Long-stamen length, sqrt(mm) & 25.24 & $<\mathbf{0 . 0 0 1}$ & 2.16 & 0.142 & $<0.001$ & 0.006 & $<0.001$ & 0.007 \\
\hline Short-stamen herkogamy, sqrt(mm) & 9.91 & 0.194 & 1.20 & 0.274 & 0.001 & 0.005 & $<0.001$ & 0.007 \\
\hline Long-stamen herkogamy, sqrt(mm) & 8.28 & 0.308 & 0.23 & 0.635 & 0.001 & 0.005 & $<0.001$ & 0.005 \\
\hline Pollen number & 18.23 & 0.011 & 4.09 & 0.043 & $<0.001$ & $80 \times 10^{5}$ & $61 \times 10^{5}$ & $41 \times 10^{7}$ \\
\hline Ovule number & 26.36 & $<0.001$ & 10.33 & 0.001 & 1.897 & 21.991 & 1.688 & 12.053 \\
\hline $\mathrm{P} / \mathrm{O}$ ratio & 28.36 & $<0.001$ & 12.14 & $<0.001$ & $<0.001$ & $47 \times 10^{2}$ & $20 \times 10^{3}$ & $54 \times 10^{4}$ \\
\hline Pollen size, $\left(\mu \mathrm{m}^{2}\right)$ & 5.06 & 0.537 & 0.07 & 0.785 & $21 \times 10$ & 65.073 & 6.043 & $53 \times 10$ \\
\hline
\end{tabular}

Traits used were: corolla area, corolla shape (dissymmetric, monosymmetric with top-bottom, and monosymmetric with left-right variation), pistil length, herkogamy levels, pollen number, ovule number, and $\mathrm{P} / \mathrm{O}$ ratio

$P$ values were based on likelihood ratio tests (LRTs), and significant effects are highlighted in bold. Random effects of population, individual plant, tray, and residual error are represented by variance estimates

appears to have evolved in clusters with and without traits closer to the selfing syndrome.

\section{The selfing syndrome in A. lyrata}

As one would expect in species with intraspecific variation for mating system, the magnitude of the changes towards the selfing syndrome in selfing populations $(9.2 \%$ smaller corolla area and $21.5 \%$ lower $\mathrm{P} / \mathrm{O}$ ratios) was small compared to differences between closely related species with contrasting mating systems. For example, the selfing Capsella rubella had a $84.7 \%$ smaller corolla and a $83.0 \%$ smaller $\mathrm{P} / \mathrm{O}$ ratio in comparison with its outcrossing relative $C$. grandiflora (Sicard et al. 2011), and the selfing Mimulus nasutus had a $85.0 \%$ smaller corolla width and a $83.1 \%$ lower pollen number than the outcrossing $M$. guttatus (Fishman et al. 2002). Even in the few systems with intraspecific mating-system variation due to the breakdown of self-incompatibility in which floral traits have been quantified, differences between outcrossing and selfing populations tended to be more pronounced. For example, corolla diameter and tube length in Abronia umbellata selfing populations were reduced by $43.0 \%$ and $54.0 \%$, respectively (Doubleday et al. 2013), petal area was reduced by $60.0 \%$ in selfing populations of Arabis alpina (Tedder et al. 2015), petal length was reduced by $37.1 \%$ in selfing populations of Camissoniopsis cheiranthifolia (Button et al. 2012), and selfers in Leavenworthia crassa had $33.3 \%$ and $27.8 \%$ smaller petal width and length (Lyons and Antonovics 1991). In Leavenworthia alabamica, which is the only species with dated intraspecific transitions to selfing (Busch et al. 2011), petal length of a 150,000-yearold selfing race was reduced by $27.0 \%$, whereas only by $8.2 \%$ in a younger, 12,000-50,000-year-old selfing race (Busch 2005). Thus, the floral changes towards the selfing syndrome in selfing populations of A. lyrata are, despite high selfing rates (Foxe et al. 2010), not as pronounced as in the other studied systems with intraspecific variation for mating system, with the exception of the younger selfing race of $L$. alabamica.

The simplest potential explanation for the relatively small floral-trait differences between outcrossing and selfing populations of North American A. lyrata is that the evolution of selfing has been, so recently that there was insufficient time to allow for larger changes in floral traits. Although the transitions to selfing have not been dated and 
might have happened before colonization of its current distribution, the spatial distribution of selfing and outcrossing populations suggests that selfing has evolved at least twice during or after colonization of the area that was covered by ice during the last Glacial Maximum, which lasted until c. 10,000 years ago (Hoebe et al. 2009; Foxe et al. 2010). This suggests that the transitions to selfing in A. lyrata and the 12,000-50,000-year-old transition to selfing in L. alabamica are of similar age, and that this explains the relatively small changes towards the selfing syndrome.

Two other potential explanations, which may or may not operate in conjunction with a recent origin, include continued dependency of selfing plants on pollinators and pleiotropic effects imposing evolutionary constraints on evolution of the selfing syndrome. In contrast to species with a clear selfing syndrome, A. lyrata plants from selfing populations do not always develop fruits from all their flowers when kept in insect-free environments (S Carleial, University of Konstanz, Germany, unpubl. results), and so are not completely autonomous selfers. This suggests that they still require vector-mediated pollen transfer for (self) pollination. Consequently, selection may still favour floral features that are important for pollinator attraction. This is surprising given that selection should strongly favour traits that allow autonomous selfing. An explanation may be that a selfing syndrome resulting in autonomous selfing cannot be selected from standing genetic variation in selfincompatible outcrossing populations, but requires a new mutation (Sicard et al. 2011; Barrett et al. 2014) and thus sufficient time to appear. Pleiotropic effects may also constrain the correlated evolution of floral traits (Ashman and Majetic 2006). For example, if the genetic make-up resulting in large flowers simultaneously results in large leaves, selection for smaller flowers may be opposed by selection for maintaining a certain leaf size. Although we cannot completely exclude these other explanations, we conclude that most evidence indicates that the relatively recent origin explains why the transitions to selfing has led to only minor changes towards the selfing syndrome, both in A. lyrata and in the younger race of L. alabamica (Busch 2005).

We found that the shift towards reduced $\mathrm{P} / \mathrm{O}$ ratios in selfing populations was proportionally larger than the reduction in floral size $(21.5 \%$ vs. $9.2 \%$; Fig. 1$)$. An even stronger pattern had emerged in L. alabamica, where the younger selfing lineage showed a reduction in $\mathrm{P} / \mathrm{O}$ ratio of $46.5 \%$, compared to $8.2 \%$ for petal length (in the older lineage, $\mathrm{P} / \mathrm{O}$ ratios had been reduced by $58.7 \%$ vs. $27.0 \%$ for petal length). Future work should test whether the differences in magnitude are related to differences in numerical properties (subtle changes in pollen or ovule number can confer large changes in $\mathrm{P} / \mathrm{O}$ ratios) or are due to selective pressures after the evolution of selfing driving resource re-allocation from male to female function (sex allocation theory; Brunet 1992) may be stronger than those operating on floral structures. The latter may be plausible, since changes towards decreased $\mathrm{P} / \mathrm{O}$ ratios likely provide a more immediate fitness advantage than other floral traits, especially if selfers still need pollinators for their pollination. There may also be more standing genetic variation for $\mathrm{P} / \mathrm{O}$ ratios than for other floral traits, since the coefficient of variation $(\mathrm{CV})$ for pollen and ovule numbers and consequently for $\mathrm{P} / \mathrm{O}$ ratios were larger than for other traits (Online Resource 6). Quantitative genetic approaches are needed to confirm whether outcrossers indeed harbour more evolutionary potential for pollen or ovule numbers than for other traits. The range of $\mathrm{P} / \mathrm{O}$ ratios we observed in selfing populations of A. lyrata (716 to 1275 ) covers both typical autogamy (mean values under 800: Alarcón et al. 2011) and outcrossing (mean values greater than 1000: Cruden 1977). This further supports the idea that insufficient time has passed to allow for the evolution of a "complete" selfing syndrome.

\section{Flower shape in A. lyrata}

In A. lyrata flowers, we found principal components representing shape variation that were similar to the ones found in E. mediohispanicum, where they were called relative warps (Gómez et al. 2006), as well as in several other species of the Brassicaceae family (Gómez et al. 2016). Interestingly, the order and the variance explained by each principal component in our analysis were consistently similar to the ones found in E. mediohispanicum (Gómez et al. 2006). As in E. mediohispanicum, the first principal component represented (dissymmetric) variation in petal parallelism, the second represented top-bottom variation in petal parallelism (monosymmetric top-bottom variation), and the third showed changes in lateral development of petals (monosymmetric left-right variation) (Fig. 2). Given the striking parallel patterns of shape variation between A. lyrata and E. mediohispanicum and Brassicaceae in general, it would be interesting to test how much of the shape variation can be attributed to genetic variation, and how much to developmental plasticity, since this has important implications for the evolvability of floral shape in Brassicaceae.

In general, for the major categories of shape variation (the first two principal components), we found no differences between outcrossing and selfing populations. For the third principal component (left-right monosymmetric variation), we found that flowers of selfing populations converged more frequently to the left from the flower's vertical axis, while flowers of outcrossing populations had the opposite tendency (Fig. 2; Online Resource 2 for details of the exact interpretation of monosymmetric left-right variation). Even in species without specialized pollination, 
different pollinators may show preferences for alternative floral shapes (Gómez et al. 2009; Gómez and Perfectti 2010; but see Galen 1999; Frey and Bukoski 2014), and a shift in pollinator requirement may-in principleexplain the observed shape difference between outcrossing and selfing populations. However, our results should be interpreted carefully, because the third principal component only accounted for $10.6 \%$ of the total shape variation (Fig. 2), and geometric morphometric analyses are very sensitive and thus prone to sampling bias (Zelditch et al. 2012). Moreover, a common garden experiment in Germany indicated no differences in pollinator visitation associated with any of the shape variation (S Carleial, University of Konstanz, Germany, unpubl. res.). Therefore, we conservatively conclude that drift or sampling bias have led to the very subtle morphological differences we observed, rather than any selective process, and that the evolution of selfing is not associated with strong changes in floral shape.

\section{Floral integration in A. lyrata}

Phenotypic integration of floral traits in A. lyrata was significant, but there were no differences between outcrossing and selfing populations. This did not support our expectation that selfing should be associated with reduced flower integration, as previously found in L. alabamica (Anderson and Busch 2006). In contrast to Anderson and Busch (2006), who also used individual plants from different populations, we accounted for non-independence of correlations within populations, and therefore, the effective sample size of our comparison was relatively low (six outcrossing and five selfing populations). Moreover, because Anderson and Busch (2006) did not account for population in their analysis, the weaker trait correlations among selfing populations that they report may not reflect lower floral-integration within populations, but rather lower correlations across populations. Our analysis does account for population, but may suffer from a lack of statistical power due to the low effective sample size produced after averaging population values.

Nevertheless, the estimates of floral integration were of very similar magnitude between outcrossing and selfing populations $(0.46 \pm 0.23$ vs. $0.45 \pm 0.35)$, which suggest that the absence of a significant effect of mating system on floral integration really indicates that the evolution of selfing in A. lyrata has not had an effect on floral integration. As explained for floral morphology above, there may simply have been insufficient time for floral integration to change in A. lyrata. However, several other factors may also explain this result. First, even if floral integration is decreased due to relaxed pollinator selection after a transition to selfing, increased homozygosity will increase linkage disequilibrium (Wright et al. 2008). Such linkage disequilibrium may prevent traits to evolve independently (Kelly 1999), and even lead to increased trait integration. A recent survey of floral integration in 64 flowering plant species concluded that selfing species actually show more floral integration than outcrossing species (Fornoni et al. 2015). Similarly, if different flower traits have a common genetic basis (i.e., pleiotropic effects; Conner 2002) responses to selection for one trait will result in simultaneous changes in other traits, and maintain floral integration. Finally, flowers might consist of several sets of integrated modules (Klingenberg 2008). Relaxed pollinator selection may lead to reduced integration of one flower module, but other selective pressures (for example, selection to maintain fecundity) may still operate to maintain floral integration at the whole flower level. In summary, it is difficult to predict whether one expects floral integration to increase or decrease after a transition to selfing, and the expectations may depend on the choice of traits.

\section{Differences in floral traits among genetic clusters}

Previous work had subdivided North American A. lyrata in western and eastern genetic clusters (Griffin and Willi 2014). Our sampling focused on the western cluster and the population genetic structure within this cluster (Foxe et al. 2010), because most known selfing populations are in this cluster. One of the selfing populations (LPT, cluster "purple", Online Resource 1) was admixed between the western and eastern clusters (Griffin and Willi 2014), and this corresponded with a remarkable floral morphology compared to the other selfing populations in our design, with relatively large flowers, pistils, and a larger P/O ratio (Fig. 1). It would, therefore, be interesting to compare the floral morphology between the western and eastern clusters.

Within the western cluster, a more fine scale clustering analysis (Foxe et al. 2010) had identified the existence of two population genetic clusters that contain both selfing and outcrossing populations (clusters "blue" and "pink"). Other clusters only contained outcrossing ("yellow") or only selfing populations ("purple" and "orange"), and two outcrossing populations were admixed. We found significant differences in floral morphology between these genetic clusters that exceeded in magnitude the overall differences due to mating system (Fig. 1; Table 1). Regardless of their mating system, the populations in the genetic clusters "pink" and "orange" had flowers with trait values significantly closer to the selfing syndrome than the populations in other clusters (Fig. 1).

These patterns suggest that the differences in flower morphology between populations have a genetic basis, which is not surprising given that flower traits in general (Bradshaw et al. 1995) and the selfing syndrome in particular has a genetic basis (Sicard et al. 2011). Furthermore, our findings 
raise the question whether clusters with floral traits closer to the selfing syndrome may be more likely to evolve selfing. This idea of pre-adaptation originates from research on invasions, in which it was shown that plants inherently possessing traits beneficial for colonizing were more prone to become invasive (Dlugosch and Parker 2007; Schlaepfer et al. 2010). Similarly, plants with smaller flowers may be pre-adapted to evolve selfing. However, we found that selfing in A. lyrata has not just evolved in possibly pre-adapted clusters, but also in clusters with floral traits closer to the outcrossing syndrome. Alongside ecological studies to identify the selective drivers of changes in floral morphology, it would be of interest to undertake quantitative genetic studies to determine the evolutionary potential and heritability for changes towards the selfing syndrome in A. lyrata. Moreover, with the genetic basis of the selfing syndrome being unravelled in C. rubella (Sicard et al. 2011), it may become possible to test whether there is a common genetic basis for the selfing syndrome in A. lyrata.

Model systems in which the transition from outcrossing to selfing is recent (i.e., systems with outcrossing and selfing populations within a species) are of great importance to improve our understanding of the processes that play a role in the evolution of the selfing syndrome. Here, we identified a system in which the (most likely very recent) evolution of selfing has merely led to subtle changes towards the selfing syndrome in comparison with other systems in which outcrossing and selfing populations have been discovered. Our detailed analyses of floral morphology further suggest that $\mathrm{P} / \mathrm{O}$ ratios may evolve faster than other traits, and that corolla shape does not change in conjunction with selfing. The stronger changes for $\mathrm{P} / \mathrm{O}$ ratio may be due to a higher standing genetic variation for this trait, or imply a larger selective pressure on traits that directly affect fecundity. We conclude that $A$. lyrata provides an excellent system to address the relative importance of evolutionary potential, pre-adaptation, and selective forces in the evolution of the selfing syndrome, ideally through a combination of quantitative genetic approaches and ecological experiments.

Acknowledgements This work was supported by the Conselho Nacional de Desenvolvimento Científico e Tecnológico (CNPq; grant number 246436/2012-3), the International Max-Planck Research School for Organismal Biology (IMPRS), and the Excellence Initiative (Independent Research Startup Grant to MS). We thank Barbara Mable for sharing seeds, Otmar Ficht and Claudia Martin for plant care, the Bioimaging Center (University of Konstanz) for help with programming and analysing in ImageJ, Christian Klingenberg and Carmelo Fruciano for help with geometric morphometrics, and Michael Lenhard, Christian Kappel and Adrien Sicard (University of Potsdam) for advice and discussion. Finally, we thank José María Gómez, all other reviewers and editors for their helpful suggestions and constructive criticism that helped a lot to improve the manuscript.

Author contribution statement MS conceived the project. SC performed the experiments, collected data and performed morphometric analyses. SC, MvK, and MS performed statistical analyses and wrote the manuscript.

\section{References}

Alarcón ML, Roquet C, Aldasoro JJ (2011) Evolution of pollen/ovule ratios and breeding system in Erodium (Geraniaceae). Syst Bot 36:661-676. doi:10.1600/036364411X583637

Al-Shehbaz IA, O'Kane SL Jr (2002) Taxonomy and phylogeny of Arabidopsis (Brassicaceae). Arabidopsis Book. doi:10.1199/ tab.0001

Anderson IA, Busch JW (2006) Relaxed pollinator-mediated selection weakens floral integration in self-compatible taxa of Leavenworthia (Brassicaceae). Am J Bot 93:860-867. doi:10.3732/ajb.93.6.860

Ashman TL, Majetic CJ (2006) Genetic constraints on floral evolution: a review and evaluation of patterns. Heredity 96:343-352. doi:10.1038/sj.hdy.6800815

Barrett SCH, Arunkumar R, Wright SI (2014) The demography and population genomics of evolutionary transitions to self-fertilization in plants. Philos Trans R Soc Lond B Biol Sci. doi:10.1098/ rstb.2013.0344

Bates D, Maechler M, Bolker B, Walker S (2014) lme4: Linear mixed-effects models using Eigen and S4. R Foundation for Statistical Computing, version 1.1-7, http://cran.r-project.org/web/ packages/lme4/index.html. Accessed 5 Aug 2016

Berg RL (1960) The ecological significance of correlation pleiades. Evolution 14:171-180. doi:10.2307/2405824

Bradshaw HD, Wilbert SM, Otto KG, Schemske DW (1995) Genetic mapping of floral traits associated with reproductive isolation in monkeyflowers (Mimulus). Nature 376:762-765. doi: $10.1038 / 376762 \mathrm{a} 0$

Brunet J (1992) Sex allocation in hermaphroditic plants. Trends Ecol Evol 7:79-84. doi:10.1016/0169-5347(92)90245-7

Busch JW (2005) The evolution of self-compatibility in geographically peripheral populations of Leavenworthia alabamica (Brassicaceae). Am J Bot 92:1503-1512. doi:10.3732/ajb.92.9.1503

Busch JW, Joly S, Schoen DJ (2011) Demographic signatures accompanying the evolution of selfing in Leavenworthia alabamica. Mol Biol Evol 28:1717-1729. doi:10.1093/molbev/msq352

Button L, Villalobos AL, Dart SR, Eckert CG (2012) Reduced petal size and color associated with transitions from outcrossing to selfing in Camissoniopsis cheiranthifolia (Onagraceae). Int $\mathbf{J}$ Plant Sci 173:251-260. doi:10.1086/663972

Cheverud JM, Wagner GP, Dow MM (1989) Methods for the comparative analysis of variation patterns. Syst Zool 38:201-213. doi: $10.2307 / 2992282$

Clauss MJ, Koch MA (2006) Poorly known relatives of Arabidopsis thaliana. Trends Plant Sci 11:449-459. doi:10.1016/j. tplants.2006.07.005

Conner JK (2002) Genetic mechanisms of floral trait correlations in a natural population. Nature 420:407-410. doi:10.1038/ Nature01105

Cruden RW (1977) Pollen-ovule ratios: a conservative indicator of breeding systems in flowering plants. Evolution 31:32-46. doi: $10.2307 / 2407542$

Dlugosch KM, Parker IM (2007) Molecular and quantitative trait variation across the native range of the invasive species Hypericum canariense: evidence for ancient patterns of colonization via pre-adaptation? Mol Ecol 16:4269-4283. doi:10.1111/j.1365-294X.2007.03508.x

Doubleday LAD, Raguso RA, Eckert CG (2013) Dramatic vestigialization of floral fragrance across a transition from outcrossing to selfing in Abronia umbellata (Nyctaginaceae). Am J Bot 100:2280-2292. doi:10.3732/ajb.1300159 
Fishman L, Kelly AJ, Willis JH, Noor M (2002) Minor quantitative trait loci underlie floral traits associated with mating system divergence in Mimulus. Evolution 56:2138-2155. doi:10.1554/0014-3820(2002)056[2138:MQTLUF]2.0.CO;2

Fornoni J, Ordano M, Pérez-Ishiwara R, Boege K, Domínguez CA (2015) A comparison of floral integration between selfing and outcrossing species: a meta-analysis. Ann Bot. doi:10.1093/aob/ mcv166 (mev166)

Foxe JP, Slotte T, Stahl EA, Neuffer B, Hurka H, Wright SI (2009) Recent speciation associated with the evolution of selfing in Capsella. Proc Natl Acad Sci USA 106:5241-5245. doi:10.1073/ pnas.0807679106

Foxe JP, Stift M, Tedder A, Haudry A, Wright SI, Mable BK (2010) Reconstructing origins of loss of self-incompatibility and selfing in North American Arabidopsis lyrata: a population genetic context. Evolution 64:3495-3510. doi:10.1111/j.1558-5646.2010.01094.x

Frey FM, Bukoski M (2014) Floral symmetry is associated with flower size and pollen production but not insect visitation rates in Geranium robertianum (Geraniaceae). Plant Species Biol 29:272-280. doi:10.1111/1442-1984.12021

Galen C (1999) Why do flowers vary? The functional ecology of variation in flower size and form within natural plant populations. Bioscience 49:631-640. doi:10.2307/1313439

Gómez JM, Perfectti F (2010) Evolution of complex traits: the case of Erysimum corolla shape. Int J Plant Sci 171:987-998. doi:10.1086/656475

Gómez JM, Perfectti F, Camacho JPM (2006) Natural selection on Erysimum mediohispanicum flower shape: insights into the evolution of zygomorphy. Am Nat 168:531-545. doi: $10.1086 / 507048$

Gómez JM, Bosch J, Perfectti F, Fernandez JD, Abdelaziz M, Camacho JP (2008) Spatial variation in selection on corolla shape in a generalist plant is promoted by the preference patterns of its local pollinators. Proc R Soc Lond B Biol Sci 275:2241-2249. doi:10.1098/rspb.2008.0512

Gómez JM, Perfectti F, Bosch J, Camacho JPM (2009) A geographic selection mosaic in a generalized plant-pollinator-herbivore system. Ecol Monogr 79:245-263. doi:10.1890/08-0511.1

Gómez JM, Torices R, Lorite J, Klingenberg CP, Perfectti F (2016) The role of pollinators in the evolution of corolla shape variation, disparity and integration in a highly diversified plant family with a conserved floral bauplan. Ann Bot 117:889-904

Griffin PC, Willi Y (2014) Evolutionary shifts to self-fertilisation restricted to geographic range margins in North American Arabidopsis lyrata. Ecol Lett 17:484-490. doi:10.1111/ele.12248

Guo YL, Bechsgaard JS, Slotte T, Neuffer B, Lascoux M, Weigel D, Schierup MH (2009) Recent speciation of Capsella rubella from Capsella grandiflora, associated with loss of self-incompatibility and an extreme bottleneck. Proc Natl Acad Sci USA 106:52465251. doi:10.1073/pnas.0808012106

Haudry A, Zha HG, Stift M, Mable BK (2012) Disentangling the effects of breakdown of self-incompatibility and transition to selfing in North American Arabidopsis lyrata. Mol Ecol 21:1130-1142. doi:10.1111/j.1365-294X.2011.05435.x

Hoebe PN (2009) Evolutionary dynamics of mating systems in populations of North American Arabidopsis lyrata. In: PhD dissertation, Faculty of Biomedical and Life Sciences, University of Glasgow, Glasgow, UK

Hoebe PN, Stift M, Tedder A, Mable BK (2009) Multiple losses of self-incompatibility in North-American Arabidopsis lyrata? Phylogeographic context and population genetic consequences. Mol Ecol 18:4924-4939. doi:10.1111/j.1365-294X.2009.04400.x

Hoebe PN, Stift M, Holub EB, Mable BK (2011) The effect of mating system on growth of Arabidopsis lyrata in response to inoculation with the biotrophic parasite Albugo candida. J Evol Biol 24:391-401. doi:10.1111/j.1420-9101.2010.02177.x

Hothorn T, Bretz F, Westfall P, Heiberger RM, Schutzenmeister A (2015) multcomp: simultaneous inference in general parametric models. R Foundation for Statistical Computing, version 1.3-9, http://cran.r-project.org/web/packages/multcomp/index.html. Accessed 5 Aug 2016

Igic B, Lande R, Kohn JR (2008) Loss of self-incompatibility and its evolutionary consequences. Int J Plant Sci 169:93-104. doi: $10.1086 / 523362$

Kearns CA, Inouye DW (1993) Techniques for pollination biologists. University Press of Colorado, Colorado

Kelly JK (1999) Response to selection in partially self-fertilizing populations II. Selection on multiple traits. Evolution 53:350-357. doi: $10.2307 / 2640772$

Klingenberg CP (2008) Morphological integration and developmental modularity. Annu Rev Ecol Evol Syst 39:115-132. doi:10.1146/ annurev.ecolsys.37.091305.110054

Klingenberg CP (2014) Studying morphological integration and modularity at multiple levels: concepts and analysis. Philos Trans R Soc B Biol Sci 369:20130249. doi:10.1098/rstb.2013.0249

Lyons EE, Antonovics J (1991) Breeding system evolution in Leavenworthia: breeding system variation and reproductive success in natural populations of Leavenworthia crassa (Cruciferae). Am J Bot 78:270-287. doi:10.2307/2445250

Mable BK, Adam A (2007) Patterns of genetic diversity in outcrossing and selfing populations of Arabidopsis lyrata. Mol Ecol 16:3565-3580. doi:10.1111/j.1365-294X.2007.03416.x

Mable BK, Robertson AV, Dart S, Di Berardo C, Witham L (2005) Breakdown of self-incompatibility in the perennial Arabidopsis lyrata (Brassicaceae) and its genetic consequences. Evolution 59:1437-1448. doi:10.1554/05-004

Pérez F, Arroyo MTK, Medel R (2007) Phylogenetic analysis of floral integration in Schizanthus (Solanaceae): does pollination truly integrate corolla traits? J Evol Biol 20:1730-1738. doi:10.1111/j.1420-9101.2007.01393.x

Core Team R (2013) R: a language and environment for statistical computing. R Foundation for Statistical Computing, Vienna, Austria

Raduski AR, Haney EB, Igic B (2012) The expression of self-incompatibility in angiosperms is bimodal. Evolution 66:1275-1283. doi:10.1111/j.1558-5646.2011.01505.x

Sandring S, Ågren J (2009) Pollinator-mediated selection on floral display and flowering time in the perennial herb Arabidopsis lyrata. Evolution 63:1292-1300. doi:10.1111/j.1558-5646.2009.00624.x

Savriama Y, Klingenberg CP (2011) Beyond bilateral symmetry: geometric morphometric methods for any type of symmetry. BMC Evol Biol 11:280. doi:10.1186/1471-2148-11-280

Savriama Y, Gómez JM, Perfectti F, Klingenberg CP (2012) Geometric morphometrics of corolla shape: dissecting components of symmetric and asymmetric variation in Erysimum mediohispanicum (Brassicaceae). New Phytol 196:945-954. doi:10.1111/j.1469-8137.2012.04312.x

Schlaepfer DR, Glattli M, Fischer M, van Kleunen M (2010) A multispecies experiment in their native range indicates pre-adaptation of invasive alien plant species. New Phytol 185:1087-1099. doi:10.1111/j.1469-8137.2009.03114.x

Shimizu KK, Tsuchimatsu T (2015) Evolution of selfing: recurrent patterns in molecular adaptation. Annu Rev Ecol Evol Syst. doi:10.1146/annurev-ecolsys-112414-054249

Sicard A, Lenhard M (2011) The selfing syndrome: a model for studying the genetic and evolutionary basis of morphological adaptation in plants. Ann Bot 107:1433-1443. doi:10.1093/aob/mcr023

Sicard A, Stacey N, Hermann K, Dessoly J, Neuffer B, Bäurle I, Lenhard M (2011) Genetics, evolution, and adaptive significance of 
the selfing syndrome in the genus Capsella. Plant Cell 23:31563171. doi:10.1105/tpc. 111.088237

Stift M, Hunter BD, Shaw B, Adam A, Hoebe PN, Mable BK (2013) Inbreeding depression in self-incompatible North-American Arabidopsis lyrata: disentangling genomic and S-locus-specific genetic load. Heredity 110:19-28. doi:10.1038/hdy.2012.49

Tedder A, Carleial S, Golębiewska M, Kappel C, Shimizu KK, Stift M (2015) Evolution of the selfing syndrome in Arabis alpina (Brassicaceae). PLoS ONE 10:e0126618. doi:10.1371/journal. pone. 0126618

Torices R, Muñoz-Pajares AJ (2016) PHENIX: phenotypic integration index. $\mathrm{R}$ foundation for statistical computing, version 1.3, http:// cran.r-project.org/web/packages/PHENIX/index.html. Accessed 5 Aug 2016

Wagner GP (1984) On the eigenvalue distribution of genetic and phenotypic dispersion matrices: evidence for a nonrandom organization of quantitative character variation. J Math Biol 21:77-95
Willi Y (2013a) The battle of the sexes over seed size: support for both kinship genomic imprinting and interlocus contest evolution. Am Nat 181:787-798. doi:10.1086/670196

Willi Y (2013b) Mutational meltdown in selfing Arabidopsis lyrata. Evolution 67:806-815. doi:10.1111/j.1558-5646.2012.01818.x

Wright SI, Ness RW, Foxe JP, Barrett SCH (2008) Genomic consequences of outcrossing and selfing in plants. Int J Plant Sci 169:105-118. doi:10.1086/523366

Zelditch ML, Swiderski DL, Sheets HD (2012) Geometric morphometrics for biologists: a primer. Academic Press, London

Zuur AF, Hilbe J, Ieno EN (2013) A beginner's guide to GLM and GLMM with R: a frequentist and Bayesian perspective for ecologists. Highland Statistics, Newburgh 